\title{
On Effectiveness of an Optimal Antiviral Bitherapy in HBV-HDV Coinfection Model
}

\author{
Omar Zakary \\ Faculty of Sciences Ben MSik, \\ P.O Box 7955 Sidi Othman, \\ Casablanca, Morocco
}

\author{
Mostafa Rachik \\ Faculty of Sciences Ben MSik, \\ P.O Box 7955 Sidi Othman, \\ Casablanca, Morocco
}

\author{
Ilias Elmouki \\ Faculty of Sciences Ben MSik, \\ P.O Box 7955 Sidi Othman, \\ Casablanca, Morocco
}

\begin{abstract}
In this work, dynamics between uninfected cells, HBV infected cells, HDV infected cells and HBV-HDV coinfected cells are studied, based on two systems of four ordinary differential equations. The two pre-validated differential systems which are considered in this paper, are respectively associated to the case when there is no infected cell proliferation of HBV, HDV and HBV-HDV coinfected populations, and to the case when there is an infected cell proliferation. Optimal control theory is applied to these two systems. Seeking to reduce the infected groups and increase the number of uninfected hepatocytes, two control functions are introduced in the two mathematical models, representing two types of treatments. In fact, the main goal of this work is to discuss the effectiveness of an antivial bitherapy that could include any inhibitor for HDV infection such as lonafarnib, with other classical treatments often used against HBV infection such as interferons, lamivudine, adefovir and entecavir. The optimal controls are characterized in terms of the optimality system, which is solved numerically using an iterative method with a progressive-regressive RungeKutta fourth order scheme with a change of several parameters.
\end{abstract}

\section{General Terms}

Hepatitis, Optimal control theory

\section{Keywords}

Hepatitis B, Hepatitis D, HBV-HDV coinfection, Pontryagin's maximum principle.

\section{INTRODUCTION}

Hepatitis commonly refers to the inflammation of the liver, caused either by a toxic substance or a virus. It is divided into five many types, namely hepatitis A, B, C, D and E. Hepatitis B Virus (HBV), Hepatitis C Virus (HCV) and Hepatitis D Virus (HDV) are commonly the cause of liver cirrhosis and cancer [1]. Post the discovery of Hepatitis B virus in 1970, extensive research has facilitated knowledge on its structure and replication mode. It is in this context a young gastroenterologist of Molinette hospital in Turin, brought to light in 1977 in hepatocyte nuclei of Italian patients chronically infected by the Hepatitis B Virus, a new antigen that the team named antigen Delta [1 5, 6, 7]. The antigen Delta was similar in structure and function to $\mathrm{HBcAg}$ (A protein of B virus capsid [20]).
However, further studies on sera of convalescent patients with hepatitis B allowed to authenticate it as different [8]. An animal model developed by the team of Rizzetto [9], and inoculations performed by volunteers [10], allowed to further advance in the knowledge about the antigen delta which was found to be a small defective virus which requires the presence of $B$ virus to replicate. HDV is considered among pathogenic viruses that could infect humans. It is estimated around 15 to 20 millions of patients infected with hepatitis are suffering from HBV-HDV coinfection [3 4, 15]. HBV-HDV coinfection occurs when an individual is infected by both HBV and HDV simultaneously, and whereas HBV-HDV superinfection occurs when HDV attacks an individual already infected with HBV. Studies suggest HDV infects around 70.000 people in the United States who have been already affected with HBV, but it had also been noted that region, has relatively low incidence rate of HDV which could be reasoned due to a low incidence rate of HBV as well as immunization provided against viral infection linked with Hepatitis B and D. Whereas, some countries in Middle east, Eastern Europe, central Africa and south America encounter high incidence rate of infection around 1.4 million and 1.9 million of people are affected with Hepatitis D [1 5]. In this article, it is suggested to follow new optimal control strategies associated with two types of inhibitors for HBV and HDV based on a HBV-HDV coinfection models developed in reference [2]. These two models have describe the interaction of HBV and HDV infection, one with and one without infected hepatocyte replication. Packer et al. in reference [2], consider lamivudine as treatment for blocking HBV reproduction, and showed that HDV infection is sustained during lamivudine treatment only if the HBV infected cell population does not become extinct. For a more general view, when any other type of available treatments is used, the subject is extended here towards an optimal control problem, by introducing into two pre-validated models, two control functions representing the inhibition of HBV and HDV infection respectively. The inhibition of each infection is incorporated by adding two control terms that represent the effectiveness of blocking both $\mathrm{B}$ and $\mathrm{D}$ viral reproduction and/or de novo infection. As an optimization criterion, an objective functional that balances the effect of minimizing the infected cells, maximizing the uninfected hepatocytes and minimizing the side effects of implementing the control treatments, is chosen. This paper is organized as follows. Section 2 presents the HDV model with and without two control terms. The objective functional and the characterizations of optimal controls are given in Section 3. Infected cell proliferation case is introduced in Section 4. Finally, Section 
5 includes numerical results of the two optimal controls and their associated optimal states, and discussion of the numerical results.

\section{MATHEMATICAL MODEL}

In an attempt to show the important role that HBV plays in HDV dynamics, Packer et al. in reference [2], start with presenting a model for HBV alone, they considered three distinct population concentrations within the liver: healthy hepatocytes (x), infected hepatocytes (y), and free HBV virions (v). The dynamics are governed by the following set of assumptions:

1 . Healthy cells are regenerated.

2. Healthy cells transition to an infected state due to infection by free virions.

3. Infected cells die at an increased rate due to infection.

4. Virions are produced by infected cells.

5. Virions die at a specified rate.

Where the production of virions and the death of virions and infected cells occur at constant per capita rates, and since the time scale of virion production is much shorter than the time scale of the hepatocytes life-cycle, Aaron Packer et al. [2] assume that the free virus population is in quasi-steady-state with the infected cell population. Thus, the free virions $v$ is proportional to the infected hepatocytes $y\left(v=\frac{\gamma}{\mu} y\right)$, then the HBV model is first simplified into a planar system, by replacing the incidence function

$$
\frac{\beta v(t) x(t)}{T(t)}
$$

by

$$
\frac{\sigma y(t) x(t)}{T(t)}
$$

where $\sigma=\frac{\beta \gamma}{\mu}$. Now, to incorporate the HDV in the model, two state variables accounting for hepatocytes infected with HDV and those coinfected with both HBV and HDV, are added. The state variables in the expanded model are

1. $x(t)$ : uninfected cells,

2. $y(t)$ : HBV only infected cells,

3. $z(t):$ HDV only infected cells,

4. $w(t)$ : coinfected cells.

Is is assumed the following transitions are possible :

1. $x \rightarrow y$ : healthy cell infected by HBV,

2. $x \rightarrow z$ : healthy cell infected by HDV,

3. $y \rightarrow w$ : HBV infected cell superinfected by HDV,

4. $z \rightarrow w$ : HDV infected cell superinfected by HBV.

These transitions are defined as the possible events of infection that can occur key-2.

\subsection{Model without treatment}

The following system of differential equations from [2] is resulted using the above assumptions,

$$
\begin{aligned}
\frac{d x}{d t} & =r x(t)\left(1-\frac{T(t)}{K}\right)-\frac{\sigma(y(t)+c w(t)) x(t)}{T(t)} \\
& -\frac{\delta w(t) x(t)}{T(t)} \\
\frac{d y}{d t} & =\frac{\sigma(y(t)+c w(t)) x(t)}{T(t)}-\frac{\delta w(t) y(t)}{T(t)}-a y(t) \\
\frac{d z}{d t} & =\frac{\delta w(t) x(t)}{T(t)}-\frac{\sigma(y(t)+c w(t)) z(t)}{T(t)}-a z(t)
\end{aligned}
$$

Table 1. Parameters description.

\begin{tabular}{|c|c|c|}
\hline Parameter & Description & Units \\
\hline \hline$r$ & Maximum proliferation rate & $d a y^{-1}$ \\
\hline$K$ & Homeostatic liver size & cells \\
\hline$a$ & Infected cell death rate & $d a y^{-1}$ \\
\hline$c$ & HBV inhibition coefficient & scalar \\
\hline$\beta$ & HBV infection rate & $d a y^{-1}$ \\
\hline$\gamma$ & HBV replication/release rate & $d a y^{-1}$ \\
\hline$\mu$ & Hepatitis B virion death rate & $d a y^{-1}$ \\
\hline$\sigma$ & HBV infection rate $\left(\frac{\gamma \beta}{\mu}\right)$ & $d a y^{-1}$ \\
\hline$\delta$ & HDV infection rate & $d a y^{-1}$ \\
\hline
\end{tabular}

$$
\frac{d w}{d t}=\frac{\sigma(y(t)+c w(t)) z(t)}{T(t)}+\frac{\delta w(t) y(t)}{T(t)}-a w(t)
$$

Where $T(t)=x(t)+y(t)+z(t)+w(t)$. The uninfected hepatocyte population is assumed to maintain itself logistically with a homeostatic carrying capacity of $\mathrm{K}$. HBV virions are produced by hepatocytes infected with HBV alone (y) and also by coinfected hepatocytes (w), although at a reduced rate in the latter cells. By considering $c$ be the degree to which HBV production is suppressed in coinfected cells, it is concluded that the number of HBV virions is proportional to $y(t)+c w(t)$. Then the susceptible host (healthy hepatocytes) cells (x) are become infected by $\mathrm{HBV}$ at a rate $\frac{\sigma(y(t)+c w(t)) x(t)}{T(t)}$ and by HDV at a rate $\frac{\delta w(t) x(t)}{T(t)}$, since HDV virions is produced only by HBV-HDV coinfected cells $w(t)$. HBV infected cells superinfected by HDV at a rate $\frac{\delta w(t) y(t)}{T(t)}$ and HDV infected cells superinfected by HBV at a rate $\frac{\sigma(y(t)+c w(t)) z(t)}{T(t)}$. HBV, HDV and HBV-HDV infected cells die at $T(t)$ a rate $a y(t), a z(t)$ and $a w(t)$ respectively. Refer to Table 1 for a complete list of parameters descriptions and units.

\subsection{Model with treatments}

In the literature, many works focus on the identification and investigation of inhibitors of HBV and HDV [13, 14] and references therein. Recently Myrcludex B, a synthetic N-acylated preS1derived lipopeptide that inhibits HBV entry in vitro and in vivo with high efficacy [14], pegylated interferon-alpha, lamivudine, entecavir and adefovir, prenylation inhibitors such as lonafarnib [16, 17, 18] and HBV entry inhibitors attract more attention as therapies in hepatitis Delta. So far, there is no exact combination therapies for chronic HBV-HDV coinfection [15]. In general, dual or triple infection with hepatitis viruses leaves more severe hepatitis and less effective interferon (IFN) response [21, 22]. Mathematical and immunological models play an important role in the study of dynamics of infections arising from viruses. In Reference [11] the authors worked on the combination of IFN and ribavirin as a therapy for HCV. The fundamental idea in this paper is a combination of two treatments, the first treatment (resp. the second treatment), which can represent any inhibitors for HBV (HDV respectively) cited above, is used to reduce the production of virions and/or the de novo rate of infection for HBV (HDV respectively). The model is represented by the following system of differential equations

$$
\begin{aligned}
\frac{d x}{d t} & =r x(t)\left(1-\frac{T(t)}{K}\right) \\
& -\frac{\left(1-k_{1} u_{1}(t)\right) \sigma(y(t)+c w(t)) x(t)}{T(t)}
\end{aligned}
$$




$$
\begin{aligned}
& -\frac{\left(1-k_{2} u_{2}(t)\right) \delta w(t) x(t)}{T(t)} \\
\frac{d y}{d t} & =\frac{\left(1-k_{1} u_{1}(t)\right) \sigma(y(t)+c w(t)) x(t)}{T(t)} \\
& -\frac{\delta w(t) y(t)}{T(t)}-a y(t) \\
\frac{d z}{d t} & =\frac{\left(1-k_{2} u_{2}(t)\right) \delta w(t) x(t)}{T(t)} \\
& -\frac{\sigma(y(t)+c w(t)) z(t)}{T(t)}-a z(t) \\
\frac{d w}{d t} & =\frac{\sigma(y(t)+c w(t)) z(t)}{T(t)}+\frac{\delta w(t) y(t)}{T(t)} \\
& -a w(t)
\end{aligned}
$$

Now, to allow for the first treatment effect in blocking the infection with $\mathrm{HBV}$, the factor $\left(1-k_{1} u_{1}(t)\right)$ is incorporated. The condition $0<k_{1}<1$ is take in consideration, to indicate that the main role of the first treatment is to block the HBV viral production in addition to having some role in blocking the de-novo infection. The effect of the second treatment for blocking the infection with HDV virus is taken into account, and then the factor $\left(1-k_{2} u_{2}(t)\right)$ is incorporated, where also $0<k_{2}<1$. For the second equation, the assumption that the HBV-infected hepatocyte is produced at the rate $\frac{\left(1-k_{1} u_{1}(t)\right) \sigma(y(t)+c w(t)) x(t)}{T(t)}$ is incorporated. Thus in the third equation the HDV-infected hepatocyte is produced at the rate $\frac{\left(1-k_{2} u_{2}(t)\right) \delta w(t) x(t)}{T(t)}$. The controls $u_{1}(t)$ and $u_{2}(t)$, are bounded, Lebesgue integrable functions. If $u_{i}=1$, the inhibition is $100 \%$ effective, whereas if $u_{i}=0$, there is no inhibition, $i=1,2$.

\section{THE OPTIMAL CONTROL PROBLEM}

Now, an optimal control problem is considered to minimize the objective functional

$$
\begin{aligned}
J\left(u_{1}, u_{2}\right) & =\int_{0}^{t_{f}}\left(-A_{0} x(t)+A_{1} y(t)+A_{2} z(t)\right. \\
& \left.+A_{3} w(t)+\frac{K_{1}}{2} u_{1}^{2}(t)+\frac{K_{2}}{2} u_{2}^{2}(t)\right) d t
\end{aligned}
$$

where $A_{0}, A_{1}, A_{2}$ and $A_{3}$ are small positive constants to keep a balance in the size of $x(t), y(t), z(t)$ and $w(t)$, respectively. The square of the control variables reflects the severity of the side effects of treatments or vaccinations [23]. The positive constants $K_{1}$ and $K_{2}$ balance the size of quadratic control terms. In fact, when drugs are administered, they produce high toxicity for the human body, which is the rationale behind taking the quadratic control terms as against linear control [12]. The reason behind considering a finite time horizon is that the treatment period is usually restricted to a limited time window. The objective of this work, is to minimize the HBV only infected cells, HDV only infected cells and the HBV-HDV coinfected cells by using possible minimal side effects of control variables $u_{1}(t)$ and $u_{2}(t)$ attempting to increase the uninfected cells. An optimal control pair $\left(u_{1}^{*}, u_{2}^{*}\right)$ is sought such that

$$
J\left(u_{1}^{*}, u_{2}^{*}\right)=\min \left\{J\left(u_{1}, u_{2}\right) \mid\left(u_{1}, u_{2}\right) \in U\right\}
$$

Where

$U=\left\{\left(u_{1}, u_{2}\right) \mid u_{1}, u_{2}\right.$ measurable, $\left.0 \leq u_{1}, u_{2} \leq 1, t \in\left[0, t_{f}\right]\right\}$
In order to find an optimal solution, first the Lagrangian and Hamiltonian are presented for the optimal control problem. In fact, the Lagrangian of the optimal problem is given by

$$
\begin{aligned}
L\left(x, y, z, w, u_{1}, u_{2}\right) & =-A_{0} x(t)+A_{1} y(t)+A_{2} z(t) \\
& +A_{3} w(t)+\frac{K_{1}}{2} u_{1}^{2}(t) \\
& +\frac{K_{2}}{2} u_{2}^{2}(t)
\end{aligned}
$$

The minimal value of the Lagrangian is sought. To accomplish this, the Hamiltonian $\mathcal{H}$ is defined as follows

$$
\begin{aligned}
\mathcal{H} & =L\left(x, y, z, w, u_{1}, u_{2}\right) \\
& +\lambda_{1}(t)\left[r x(t)\left(1-\frac{T(t)}{K}\right)\right. \\
& -\frac{\left(1-k_{1} u_{1}(t)\right) \sigma(y(t)+c w(t)) x(t)}{T(t)} \\
& \left.-\frac{\left(1-k_{2} u_{2}(t)\right) \delta w(t) x(t)}{T(t)}\right] \\
& +\lambda_{2}(t)\left[\frac{\left(1-k_{1} u_{1}(t)\right) \sigma(y(t)+c w(t)) x(t)}{T(t)}-\frac{\delta w(t) y(t)}{T(t)}\right. \\
& -a y(t)]+\lambda_{3}(t)\left[\frac{\left(1-k_{2} u_{2}(t)\right) \delta w(t) x(t)}{T(t)}\right. \\
& \left.-\frac{\sigma(y(t)+c w(t)) z(t)}{T(t)}-a z(t)\right] \\
& +\lambda_{4}(t)\left[\frac{\sigma(y(t)+c w(t)) z(t)}{T(t)}+\frac{\delta w(t) y(t)}{T(t)}-a w(t)\right] \quad(11)
\end{aligned}
$$

To find the optimal solution, the Pontryagin's Maximum Principle is applied to the Hamiltonian [24], and the following theorem is obtained. Let $x^{*}(t), y^{*}(t), z^{*}(t)$ and $w^{*}(t)$ be optimal state solutions with associated optimal control variables $u_{1}^{*}(t)$ and $u_{2}^{*}(t)$ for the optimal control problem (9). Then, there exist adjoint variables $\lambda_{1}(t), \lambda_{2}(t), \lambda_{3}(t)$ and $\lambda_{4}(t)$ that satisfy

$$
\begin{aligned}
\dot{\lambda}_{1}(t) & =-\left[-A_{0}+r\left(1-\frac{T(t)}{K}\right) \lambda_{1}(t)\right. \\
& +\frac{\left(1-k_{1} u_{1}^{*}(t)\right) \sigma\left(y^{*}(t)+c w^{*}(t)\right)}{T(t)}\left(\lambda_{2}(t)-\lambda_{1}(t)\right) \\
& \left.+\frac{\left(1-k_{2} u_{2}^{*}(t)\right) \delta w^{*}(t)}{T(t)}\left(\lambda_{3}(t)-\lambda_{1}(t)\right)\right] \\
\dot{\lambda}_{2}(t) & =-\left[A_{1}-a \lambda_{2}(t)+\left(\lambda_{2}(t)-\lambda_{1}(t)\right) \frac{\left(1-k_{1} u_{1}^{*}(t)\right) \sigma x^{*}(t)}{T(t)}\right. \\
& \left.+\left(\lambda_{4}(t)-\lambda_{2}(t)\right) \frac{\delta w^{*}(t)}{T(t)}+\left(\lambda_{4}(t)-\lambda_{3}(t)\right) \frac{\sigma z^{*}(t)}{T(t)}\right] \\
\dot{\lambda}_{3}(t) & =-\left[A_{2}-a \lambda_{3}(t)+\left(\lambda_{4}(t)-\lambda_{3}(t)\right) \frac{\sigma\left(y^{*}(t)+c w^{*}(t)\right)}{T(t)}\right] \\
\dot{\lambda}_{4}(t) & =-\left[A_{3}-a \lambda_{4}(t)+\left(\lambda_{2}(t)-\lambda_{1}(t)\right) \frac{\left(1-k_{1} u_{1}^{*}(t)\right) \sigma c x^{*}(t)}{T(t)}\right. \\
& +\left(\lambda_{3}(t)-\lambda_{1}(t)\right) \frac{\left(1-k_{2} u_{2}^{*}(t)\right) \delta x^{*}(t)}{T(t)} \\
& \left.+\left(\lambda_{4}(t)-\lambda_{2}(t)\right) \frac{\delta y^{*}(t)}{T(t)}+\left(\lambda_{4}(t)-\lambda_{3}(t)\right) \frac{\sigma c z^{*}(t)}{T(t)}\right]
\end{aligned}
$$


with the transversality conditions $\lambda_{i}\left(t_{f}\right)=0, i=1,2,3,4$. Furthermore, the optimal controls $u_{1}^{*}(t)$ and $u_{2}^{*}(t)$ are given by

$$
\begin{aligned}
& u_{1}^{*}(t)=\max \left\{\min \left\{\left(\lambda_{2}(t)-\lambda_{1}(t)\right) \frac{k_{1} \sigma(y(t)+c w(t)) x(t)}{K_{1} T(t)}, 1\right\}, 0\right\} \\
& u_{2}^{*}(t)=\max \left\{\min \left\{\left(\lambda_{3}(t)-\lambda_{1}(t)\right) \frac{k_{2} \delta w(t) x(t)}{K_{2} T(t)}, 1\right\}, 0\right\}
\end{aligned}
$$

PROOF. To determine the adjoint equations and the transversality conditions, the Hamiltonian $\mathcal{H}$ defined by $(11)$ is used. From setting $x(t)=x^{*}(t), y(t)=y^{*}(t), z(t)=z^{*}(t)$ and $w(t)=w^{*}(t)$, and differentiating $\mathcal{H}$ with respect to $x(t), y(t), z(t)$ and $w(t)$, the following adjoint differential system is obtained

$$
\begin{aligned}
\dot{\lambda}_{1}(t)=-\frac{d \mathcal{H}}{d x} & =-\left[-A_{0}+r\left(1-\frac{T(t)}{K}\right) \lambda_{1}(t)\right. \\
& +\frac{\left(1-k_{1} u_{1}(t)\right) \sigma(y(t)+c w(t))}{T(t)}\left(\lambda_{2}(t)-\lambda_{1}(t)\right) \\
& \left.+\frac{\left(1-k_{2} u_{2}(t)\right) \delta w(t)}{T(t)}\left(\lambda_{3}(t)-\lambda_{1}(t)\right)\right] \\
\dot{\lambda}_{2}(t)=-\frac{d \mathcal{H}}{d y} & =-\left[A_{1}-a \lambda_{2}(t)\right. \\
& +\left(\lambda_{2}(t)-\lambda_{1}(t)\right) \frac{\left(1-k_{1} u_{1}(t)\right) \sigma x(t)}{T(t)} \\
& \left.+\left(\lambda_{4}(t)-\lambda_{2}(t)\right) \frac{\delta w(t)}{T(t)}+\left(\lambda_{4}(t)-\lambda_{3}(t)\right) \frac{\sigma z(t)}{T(t)}\right] \\
\dot{\lambda}_{3}(t)=-\frac{d \mathcal{H}}{d z} & =-\left[A_{2}-a \lambda_{3}(t)\right. \\
& \left.+\left(\lambda_{4}(t)-\lambda_{3}(t)\right) \frac{\sigma(y(t)+c w(t))}{T(t)}\right] \\
\dot{\lambda}_{4}(t)=-\frac{d \mathcal{H}}{d w} & =-\left[A_{3}-a \lambda_{4}(t)\right. \\
& +\left(\lambda_{2}(t)-\lambda_{1}(t)\right) \frac{\left(1-k_{1} u_{1}(t)\right) \sigma c x(t)}{T(t)} \\
& +\left(\lambda_{3}(t)-\lambda_{1}(t)\right) \frac{\left(1-k_{2} u_{2}(t)\right) \delta x(t)}{T(t)} \\
& \left.+\left(\lambda_{4}(t)-\lambda_{2}(t)\right) \frac{\delta y(t)}{T(t)}+\left(\lambda_{4}(t)-\lambda_{3}(t)\right) \frac{\sigma c z(t)}{T(t)}\right]
\end{aligned}
$$

By the optimality conditions, the derivative of $\mathcal{H}$ with respect to $u_{1}$ and $u_{2}$ are:

$$
\begin{gathered}
\frac{d \mathcal{H}}{d u_{1}}=K_{1} u_{1}(t)+\left(\lambda_{1}(t)-\lambda_{2}(t)\right) \frac{k_{1} \sigma(y(t)+c w(t)) x(t)}{T(t)}=0 \\
\Rightarrow u_{1}^{*}(t)=\left(\lambda_{2}(t)-\lambda_{1}(t)\right) \frac{k_{1} \sigma(y(t)+c w(t)) x(t)}{K_{1} T(t)} \\
\frac{d \mathcal{H}}{d u_{2}}=K_{2} u_{2}(t)+\left(\lambda_{1}(t)-\lambda_{3}(t)\right) \frac{k_{2} \delta w(t) x(t)}{T(t)}=0 \\
\Rightarrow u_{2}^{*}(t)=\left(\lambda_{3}(t)-\lambda_{1}(t)\right) \frac{k_{2} \delta w(t) x(t)}{K_{2} T(t)}
\end{gathered}
$$

Since the controls are bounded below by 0 and bounded above by 1 , thus their characterizations are given by:

$u_{1}^{*}(t)=\max \left\{\min \left\{\left(\lambda_{2}(t)-\lambda_{1}(t)\right) \frac{k_{1} \sigma(y(t)+c w(t)) x(t)}{K_{1} T(t)}, 1\right\}, 0\right\}$ $u_{2}^{*}(t)=\max \left\{\min \left\{\left(\lambda_{3}(t)-\lambda_{1}(t)\right) \frac{k_{2} \delta w(t) x(t)}{K_{2} T(t)}, 1\right\}, 0\right\}$

\section{MODEL WITH INFECTED CELL PROLIFERATION}

In this section, the case where the infected cells proliferate and transmit the infection vertically to daughter cells, is investigated by assuming that infected cells often contain multiple copies of the viral genome [2] and references therein. As a simple extension of system (69) which uses the logistic function for proliferation of both healthy and infected cells. The model is now given by

$$
\begin{aligned}
\frac{d x}{d t} & =r x(t)\left(1-\frac{T(t)}{K}\right)-\frac{\left(1-k_{1} u_{1}(t)\right) \sigma(y(t)+c w(t)) x(t)}{T(t)} \\
& -\frac{\left(1-k_{2} u_{2}(t)\right) \delta w(t) x(t)}{T(t)} \\
\frac{d y}{d t} & =r y(t)\left(1-\frac{T(t)}{K}\right)+\frac{\left(1-k_{1} u_{1}(t)\right) \sigma(y(t)+c w(t)) x(t)}{T(t)} \\
& -\frac{\delta w(t) y(t)}{T(t)}-a y(t) \\
\frac{d z}{d t} & =r w(t)\left(1-\frac{T(t)}{K}\right)+\frac{\left(1-k_{2} u_{2}(t)\right) \delta w(t) x(t)}{T(t)} \\
& -\frac{\sigma(y(t)+c w(t)) z(t)}{T(t)}-a z(t) \\
\frac{d w}{d t} & =r z(t)\left(1-\frac{T(t)}{K}\right)+\frac{\sigma(y(t)+c w(t)) z(t)}{T(t)}+\frac{\delta w(t) y(t)}{T(t)} \\
& -a w(t)
\end{aligned}
$$

with liver regeneration sustained by both healthy and infected cells. Note that in the third equation $(\mathrm{z})$, the term $r w(t)\left(1-\frac{T(t)}{K}\right)$ is incorporated, while in the fourth equation (w), the term $r z(t)(1-$ $\left.\frac{T(t)}{K}\right)$ is incorporated, based on the fact that the free HDV particles can only be produced by coinfected cells, and the number of HDV virions is proportional to the number of coinfected cells [2].

The objective functional is defined by:

$$
\begin{aligned}
J\left(u_{1}, u_{2}\right) & =\int_{0}^{t_{f}}\left(-A_{0} x(t)+A_{1} y(t)+A_{2} z(t)+A_{3} w(t)\right. \\
& \left.+\frac{K_{1}}{2} u_{1}^{2}(t)+\frac{K_{2}}{2} u_{2}^{2}(t)\right) d t
\end{aligned}
$$

An optimal control pair $\left(u_{1}^{*}, u_{2}^{*}\right)$ is sought such that

$$
J\left(u_{1}^{*}, u_{2}^{*}\right)=\min \left\{J\left(u_{1}, u_{2}\right) \mid\left(u_{1}, u_{2}\right) \in U\right\}
$$

Where

$$
U=\left\{\left(u_{1}, u_{2}\right) \mid u_{1}, u_{2} \text { measurable, } 0 \leq u_{1}, u_{2} \leq 1, t \in\left[0, t_{f}\right]\right\}
$$

As above, first the Lagrangian and Hamiltonian for the optimal control problem, are presented. In fact, the Lagrangian of the optimal problem remains

$$
\begin{aligned}
L\left(x, y, z, w, u_{1}, u_{2}\right) & =-A_{0} x(t)+A_{1} y(t)+A_{2} z(t) \\
& +A_{3} w(t)+\frac{K_{1}}{2} u_{1}^{2}(t) \\
& +\frac{K_{2}}{2} u_{2}^{2}(t)
\end{aligned}
$$



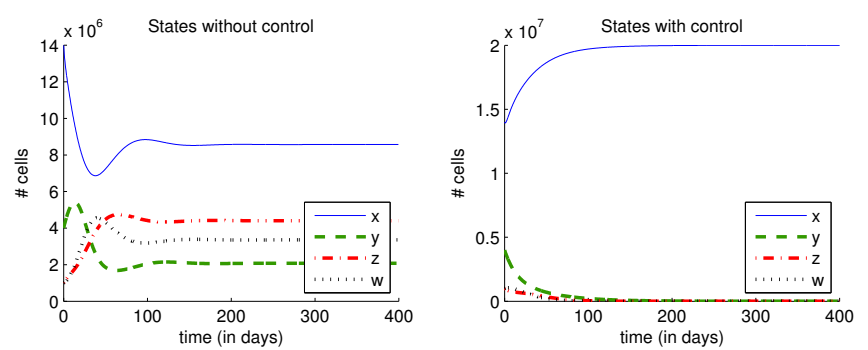

Fig. 1. Comparison between states $\mathrm{x}, \mathrm{y}, \mathrm{z}$ and $\mathrm{w}$ of systems 2,4 ) and 69 , before and after treatment respectively with $R_{b}=2.5, R_{d}=4, k_{1}=0.8$, $k_{2}=0.8$.

By same way as before, the minimal value of the Lagrangian is sought. To accomplish this as it was done above, the Hamiltonian $\mathcal{H}$ is defined as follows

$$
\begin{aligned}
\mathcal{H} & =L\left(x, y, z, w, u_{1}, u_{2}\right)+\lambda_{1}(t)\left[r x(t)\left(1-\frac{T(t)}{K}\right)\right. \\
& -\frac{\left(1-k_{1} u_{1}(t)\right) \sigma(y(t)+c w(t)) x(t)}{T(t)} \\
& \left.-\frac{\left(1-k_{2} u_{2}(t)\right) \delta w(t) x(t)}{T(t)}\right]+\lambda_{2}(t)\left[r y(t)\left(1-\frac{T(t)}{K}\right)\right. \\
& +\frac{\left(1-k_{1} u_{1}(t)\right) \sigma(y(t)+c w(t)) x(t)}{T(t)} \\
& \left.-\frac{\delta w(t) y(t)}{T(t)}-a y(t)\right]+\lambda_{3}(t)\left[r w(t)\left(1-\frac{T(t)}{K}\right)\right. \\
& \left.+\frac{\left(1-k_{2} u_{2}(t)\right) \delta w(t) x(t)}{T(t)}-\frac{\sigma(y(t)+c w(t)) z(t)}{T(t)}-a z(t)\right] \\
& +\lambda_{4}(t)\left[r z(t)\left(1-\frac{T(t)}{K}\right)+\frac{\sigma(y(t)+c w(t)) z(t)}{T(t)}\right. \\
& \left.+\frac{\delta w(t) y(t)}{T(t)}-a w(t)\right]
\end{aligned}
$$

Let $x^{*}(t), y^{*}(t), z^{*}(t)$ and $w^{*}(t)$ be optimal state solutions with associated optimal control variables $u_{1}^{*}(t)$ and $u_{2}^{*}(t)$ for the optimal control problem (16. Then, there exist adjoint variables $\lambda_{1}(t), \lambda_{2}(t), \lambda_{3}(t)$ and $\bar{\lambda}_{4}(t)$ that satisfy

$$
\begin{aligned}
\dot{\lambda}_{1}(t) & =-\left[-A_{0}+r\left(1-\frac{T(t)}{K}\right) \lambda_{1}(t)\right. \\
& +\frac{\left(1-k_{1} u_{1}^{*}(t)\right) \sigma\left(y^{*}(t)+c w^{*}(t)\right)}{T(t)}\left(\lambda_{2}(t)-\lambda_{1}(t)\right) \\
& \left.+\frac{\left(1-k_{2} u_{2}^{*}(t)\right) \delta w^{*}(t)}{T(t)}\left(\lambda_{3}(t)-\lambda_{1}(t)\right)\right] \\
\dot{\lambda}_{2}(t) & =-\left[A_{1}+r\left(1-\frac{T(t)}{K}\right) \lambda_{2}(t)-a \lambda_{2}(t)\right. \\
& +\left(\lambda_{2}(t)-\lambda_{1}(t)\right) \frac{\left(1-k_{1} u_{1}^{*}(t)\right) \sigma x^{*}(t)}{T(t)} \\
& \left.+\left(\lambda_{4}(t)-\lambda_{2}(t)\right) \frac{\delta w^{*}(t)}{T(t)}+\left(\lambda_{4}(t)-\lambda_{3}(t)\right) \frac{\sigma z^{*}(t)}{T(t)}\right] \\
\dot{\lambda}_{3}(t) & =-\left[A_{2}+r\left(1-\frac{T(t)}{K}\right) \lambda_{4}(t)-a \lambda_{3}(t)\right.
\end{aligned}
$$
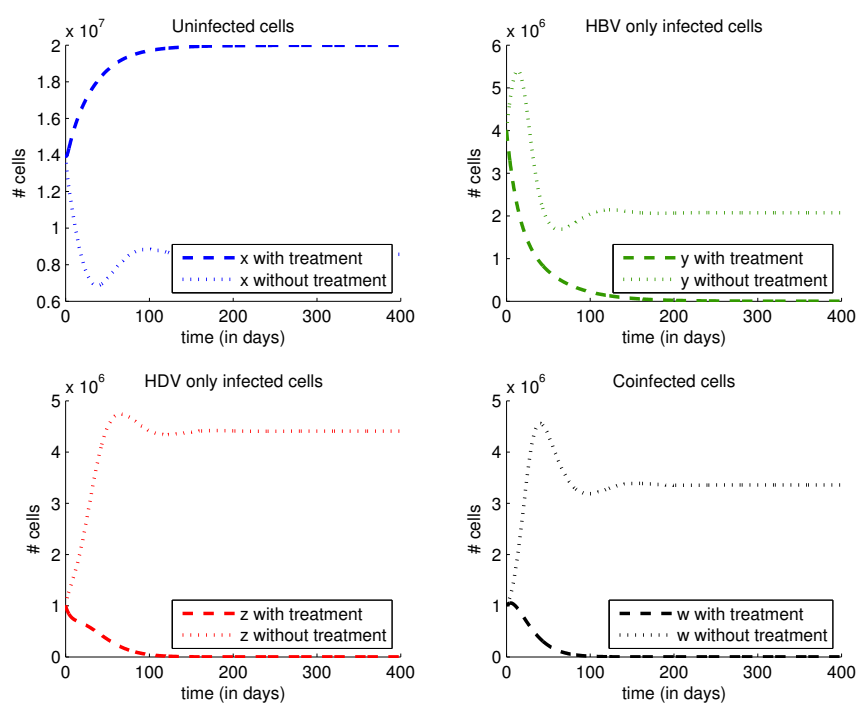

Fig. 2. Comparison between states $\mathrm{x}, \mathrm{y}, \mathrm{z}$ and $\mathrm{w}$ of systems (2,4) and $(6$ 9 before and after treatment respectively with $k_{1}=0.8, k_{2}=0.8, \delta=$ $0.2772, \sigma=0.17325, R_{b}=2.5, R_{d}=4$

$$
\begin{aligned}
& \left.+\left(\lambda_{4}(t)-\lambda_{3}(t)\right) \frac{\sigma\left(y^{*}(t)+c w^{*}(t)\right)}{T(t)}\right] \\
\dot{\lambda}_{4}(t) & =-\left[A_{3}+r\left(1-\frac{T(t)}{K}\right) \lambda_{3}(t)-a \lambda_{4}(t)\right. \\
& +\left(\lambda_{2}(t)-\lambda_{1}(t)\right) \frac{\left(1-k_{1} u_{1}^{*}(t)\right) \sigma c x^{*}(t)}{T(t)} \\
& +\left(\lambda_{3}(t)-\lambda_{1}(t)\right) \frac{\left(1-k_{2} u_{2}^{*}(t)\right) \delta x^{*}(t)}{T(t)} \\
& \left.+\left(\lambda_{4}(t)-\lambda_{2}(t)\right) \frac{\delta y^{*}(t)}{T(t)}+\left(\lambda_{4}(t)-\lambda_{3}(t)\right) \frac{\sigma c z^{*}(t)}{T(t)}\right]
\end{aligned}
$$

with the transversality conditions $\lambda_{i}\left(t_{f}\right)=0, i=1,2,3$, 4. Furthermore, the optimal controls $u_{1}^{*}(t)$ and $u_{2}^{*}(t)$ are given by

$$
\begin{aligned}
& u_{1}^{*}(t)=\max \left\{\min \left\{\left(\lambda_{2}(t)-\lambda_{1}(t)\right) \frac{k_{1} \sigma(y(t)+c w(t)) x(t)}{K_{1} T(t)}, 1\right\}, 0\right\} \\
& u_{2}^{*}(t)=\max \left\{\min \left\{\left(\lambda_{3}(t)-\lambda_{1}(t)\right) \frac{k_{2} \delta w(t) x(t)}{K_{2} T(t)}, 1\right\}, 0\right\}
\end{aligned}
$$

The states $\mathrm{x}, \mathrm{y}, \mathrm{z}$ and $\mathrm{w}$ and the adjoint states $\lambda_{1}, \lambda_{2}, \lambda_{3}$ and $\lambda_{4}$ are a priori $L^{\infty}$ - bounded which make the right-hand side of each equation in the two differential systems (6)-(9) and (12)- $(15)$ Lipschitz in those solutions and based on this property the uniqueness of the two controls $u_{1}^{*}$ and $u_{2}^{*}$ is proven for a small final time. Concerning the existence of the optimal controls $u_{1}^{*}$ and $u_{2}^{*}$, can directly be deduced from the compactness of $\mathrm{U}$ and the state space with the convexity of the integrand of $\mathrm{J}[19]$.

\section{NUMERICAL SIMULATIONS AND DISCUSSIONS}

Numerical simulations associated with the two optimal systems derived from the two previous mathematical models, are presented in this section. A code is written in MATLAB ${ }^{T M}$ and simulated the results using data from the article by Packer et al. [2]. The optimal- 

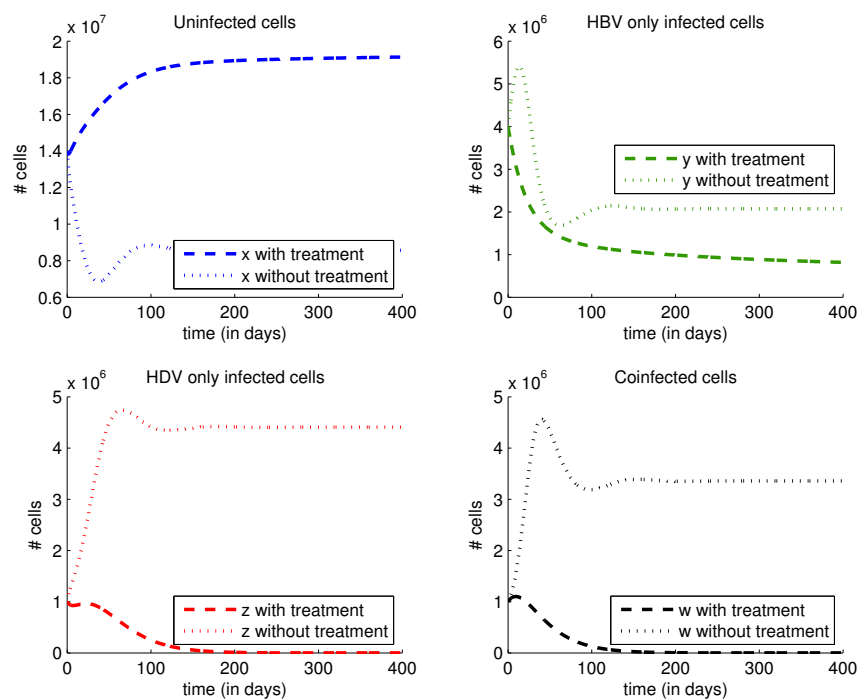

Fig. 3. Comparison between states $\mathrm{x}, \mathrm{y}, \mathrm{z}$ and $\mathrm{w}$ of systems $(24)$ and 6 9 before and after treatment respectively with $k_{1}=0.69, k_{2}=0.69$, $\delta=0.2772, \sigma=0.17325, R_{b}=2.5, R_{d}=4$

ity systems is solved using an iterative method with a progressiveregressive Runge-Kutta fourth order scheme. Such numerical procedures, are called forward-backward sweep methods, where the state system with an initial guess is solved forward in time and then the adjoint system is solved backward in time. First, starting with an initial guess for the adjoint variables $\lambda_{1}, \lambda_{2}, \lambda_{3}$ and $\lambda_{4}$, the state equations are solved by a forward Runge-Kutta fourth order procedure in time. Then, those state values are used to solve the adjoint equations by a backward Runge-Kutta fourth order procedure because of the transversality conditions [25] [26]. Afterwards, the optimal controls values are updated using the values of state and costate variables obtained in the previous steps. Finally, the previous steps are executed till a tolerance criterion is reached. It is observed in the simulations the effect of the optimal treatments on the viral load during the treatment period. For the figures presented here, it is assumed that the weight factor $K_{1}$ associated with control $u_{1}$ is greater or equal to $K_{2}$ which is associated with control $u_{2}$. This assumption is based on following facts: The hepatitis delta virus (HDV) is a dependent satellite virus of hepatitis B virus (HBV). While HDV is capable of copying its genome in great numbers in infected hepatocytes, it requires the hepatitis B surface antigen ( $\mathrm{HBsAg}$ ) in order to create new free virus particles [2, 15, 21, 22]. In simulations, the data used were $c=0.3$, and $a=0.0693$, and the notation $R_{b}=\frac{\sigma}{a}$ and $R_{d}=\frac{\delta}{a}$ [2] are both used, in order to show the effectiveness of the results and to simplify the comparison with results obtained in [2].

First case : $R_{b}=2.5, R_{d}=4, k_{1}=0.8, k_{2}=0.8$

Figure 1 depicts simulations of states $\mathrm{x}, \mathrm{y}, \mathrm{z}$ and $\mathrm{w}$ in the absence of treatment and the presence of treatment. Due to infection with $\mathrm{HBV}$ and HDV, and due to the absence of treatment, the simulations on left, show that $\mathrm{x}$ cells taper to a stable value around $8.5 \times 10^{6}$ c.f.u. (colony forming unit) while y, $\mathrm{z}$, w cells grow towards values around $5.5 \times 10^{6}$ c.f.u., $4.8 \times 10^{6}$ c.f.u., $4.4 \times 10^{6}$ c.f.u. respectively between 20 and 80 days of the infection period, and they stabilize in important infected cells with counts around $2 \times 10^{6}$ c.f.u., $4.2 \times 10^{6}$ c.f.u. and $3.5 \times 10^{6}$ c.f.u. respectively. The effectiveness of the treatments represented by both control functions
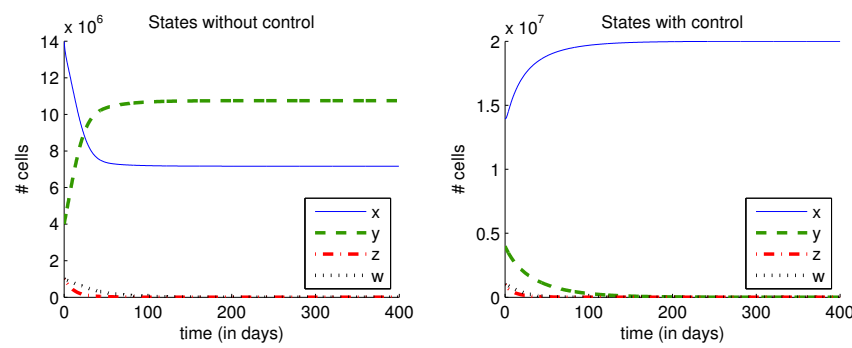

Fig. 4. Comparison between states $\mathrm{x}, \mathrm{y}, \mathrm{z}$ and $\mathrm{w}$ of systems (2,4) and 6 . 9 before and after treatment respectively with $k_{1}=0.8, k_{2}=0.8, \delta=$ $0.04158, \sigma=0.17325, R_{b}=2.5, R_{d}=0.6$

$u_{1}$ and $u_{2}$ is proved on the simulations on right of Figure 1. Since all infected cells taper off after 100 to 115 days of treatment while uninfected cells increases towards $2 \times 10^{7}$ c.f.u. widely more important than the initial condition taken by the variable $\mathrm{x}$ in both simulations on right and left. Figures 2 and 3 report some similarities from numerical results obtained in Figure 1 but with a larger scale to show exactly what happens to $\mathrm{x}, \mathrm{y}, \mathrm{z}$ and $\mathrm{w}$ cells when a treatment is used against HBV infection, HDV infection, and HBVHDV coinfection. On the other hand, it is recalled that $k_{1}$ and $k_{2}$ are used to show respectively the main roles of treatments used against HBV and HDV infection in attempt to block the viral production of B and Delta viruses, such considerations were also used by Chakrabarty et al. for the optimization of a bitherapy with interferon and ribavirin against hepatitis $\mathrm{C}[11]$. In fact, when $k_{1}$ and $k_{2}$ take values close to $1 ; k_{1}=0.8$ and $k_{2}=0.8$ in Figures 1 and 2 for instance, a rapid decrease of infected cells with HBV or HDV or even with both viral infections, is observed, and it is also seen a rapid increase of uninfected cells compared to the case where $k_{1}$ and $k_{2}$ become to have farther values to, $k_{1}=0.69$ and $k_{2}=0.69$ in Figure 4 for instance, and where $\mathrm{z}$ and $\mathrm{w}$ cells late to decrease and taper off respectively until 150 and 130 days of treatment. With regards to y cells, they only stabilize towards nonzero and minimal value around $10^{6}$ c.f.u. The uninfected cells grow towards an important amount around $1.9 \times 10^{6}$ c.f.u. but x cells count remains smaller than cases studied in previous figures. Figure 7 shows the shapes of both controls $u_{1}$ and $u_{2}$ when $k_{1}$ and $k_{2}$ change their values from 0.8 "case (a)" to 0.69 "case (b)". It is deduced from the four simulations obtained in Figure 7, the only value that changes is the one related to the second control $u_{2}$, and that shows a decrease of the value of treatment effectiveness when it comes close to 300 days and taper off in the final phase of therapy in case (a), while it conserves its value from 1 along the treatment period and takes zero value at the fixed final time $t_{f}=400$ days in case (b). The first control function $u_{1}$ takes same values of $u_{2}$ of case (b) in both cases (a) and (b). In case (a), it could be recommended now to use a treatment against HDV infection for only 300 days instead of 400 days, while using a dosage amount for HBV infection by its maximal value along the treatment period.

Second case : $R_{b}=2.5, R_{d}=0.6, k_{1}=0.8, k_{2}=0.8$

In Figure 4, on left, and in Figure 5, it is observed that states $\mathrm{z}$ and $\mathrm{w}$ in the absence of treatment stabilize in zero values between 40 and 80 days of the infection period respectively although no treatment is used yet. However, it is deduced that such results occur due to the new value of HDV reproduction number of Delta viruses related to the value of the constant delta that is smaller here " $R_{d}=0.6$ " than the value considered in the first case " $R_{d}=4$ " while a constant number of reproduction of $B$ viruses is always sufficient in participating to a decrease of uninfected cells $\mathrm{x}$ and an increase of $\mathrm{y}$ cells 

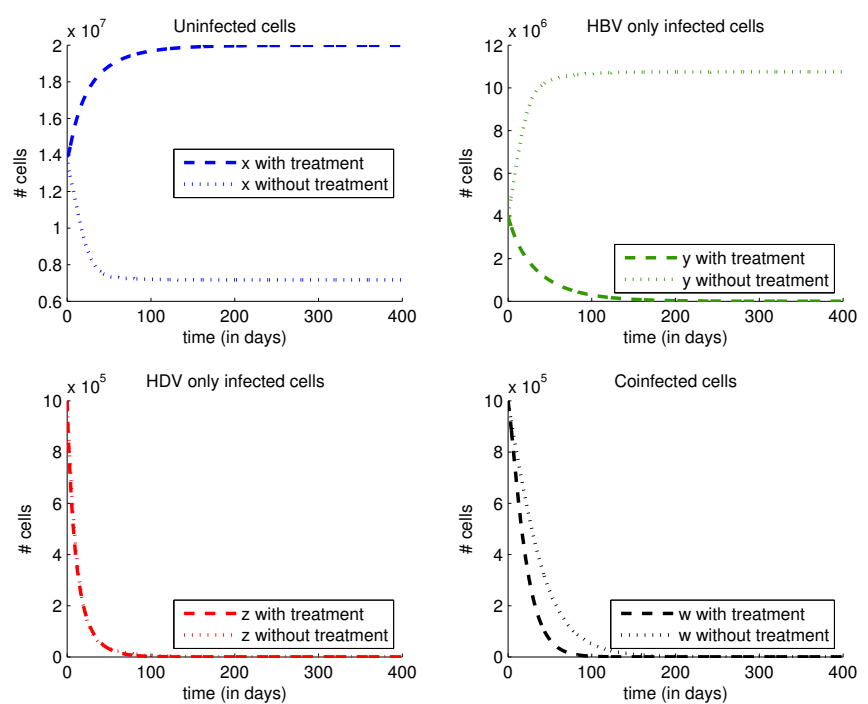

Fig. 5. Comparison between states $\mathrm{x}, \mathrm{y}, \mathrm{z}$ and $\mathrm{w}$ of systems 24 and 6 . 9] before and after treatment respectively with $k_{1}=0.8, k_{2}=0.8, \delta=$ $0.04158, \sigma=0.17325, R_{b}=2.5, R_{d}=0.6$
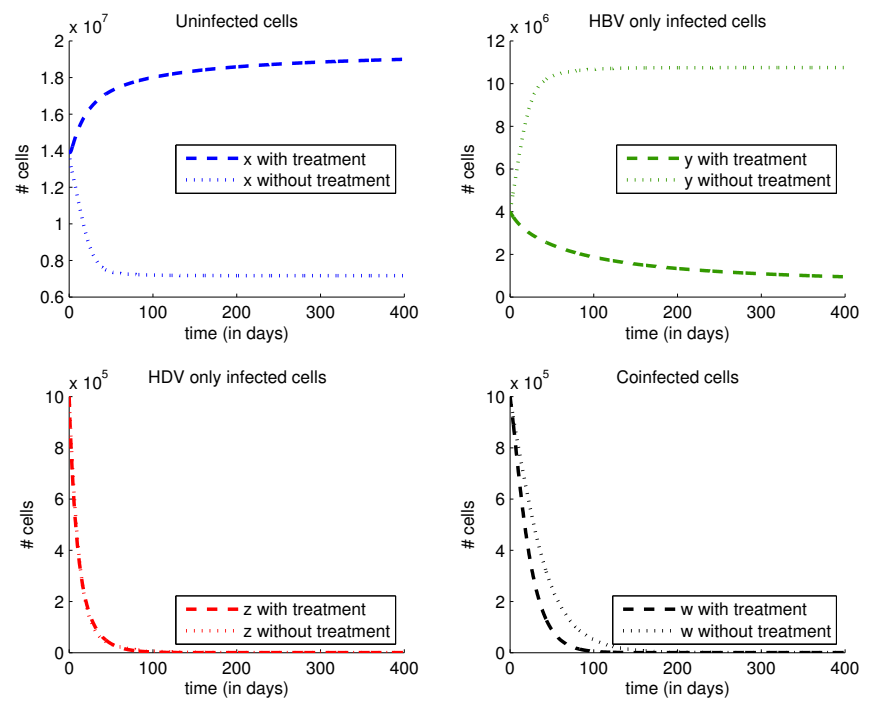

Fig. 6. Comparison between states $\mathrm{x}, \mathrm{y}, \mathrm{z}$ and $\mathrm{w}$ of systems 24 and 6 9 before and after treatment respectively with $k_{1}=0.69, k_{2}=0.69$, $\delta=0.04158, \sigma=0.17325, R_{b}=2.5, R_{d}=0.6$

as it is showed in the same figure on left. In fact, when $u_{1}$ and $u_{2}$ are zero, the uninfected cells taper to the value $0.75 \times 10^{7}$ c.f.u. after only 40 days of infection period, and when $u_{1}$ and $u_{2}$ are nonzero with $k_{1}$ and $k_{2}$ both taking the value 0.8 , uninfected cells count becomes more important and increase to the value $2 \times 10^{7}$ c.f.u. $\mathrm{z}$ cells taper off after 80 days in both cases, with and without control due the small value of $R_{d}$ in this case, while y cells taper off when they come close to the 150th day of treatment as opposite to the case when there was no treatment yet and they were increasing towards the value $10.5 \times 10^{6}$. With regards to w cells, they late to taper off for 150 days of the infection period, but rapidly stabilize in zero values in the presence of the HBV-HDV bitherapy for only 80 (a)
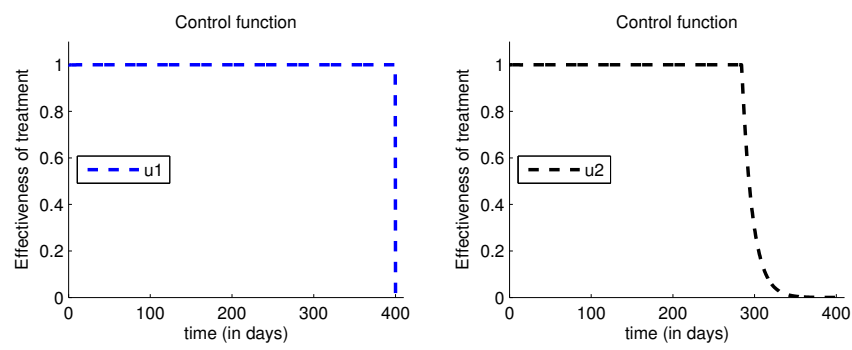

(b)
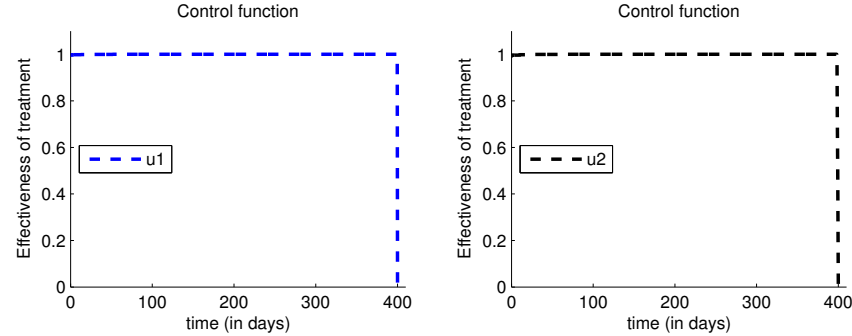

Fig. 7. Control functions with $R_{b}=2.5, R_{d}=4$. (a) $k_{1}=0.8, k_{2}=$ 0.8. (b) $k_{1}=0.69, k_{2}=0.69$.

(a)
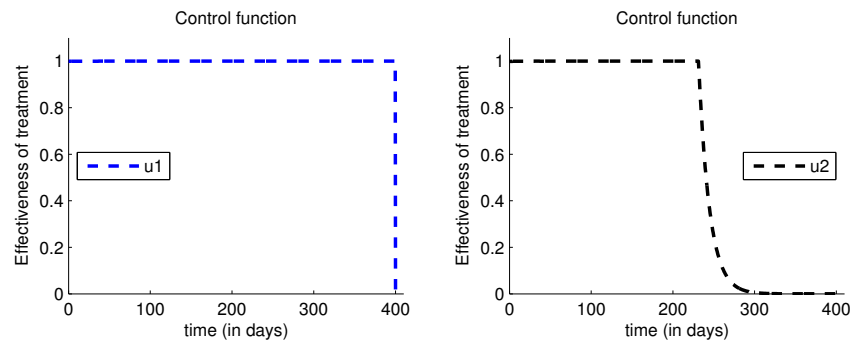

(b)
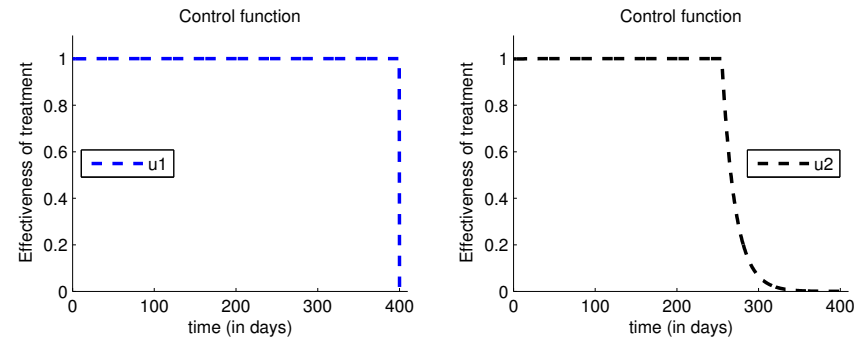

Fig. 8. Control functions with $R_{b}=2.5, R_{d}=0.6$. (a) $k_{1}=0.9$, $k_{2}=0.9$. (b) $k_{1}=0.69, k_{2}=0.69$.

days. In Figure 6, when the value 0.69 is considered for both coefficients $k_{1}$ and $k_{2}$ that are respectively associated to both controls $u_{1}$ and $u_{2}$, it is observed that uninfected cells stabilize approximately in the same value of the case where $k_{1}$ and $k_{2}$ took the value $0.8, \mathrm{z}$ and $w$ cells do not change either but y cells do not taper off in that new case but only decreases progressively to stabilize in a nonzero value that equals to $10^{6}$ c.f.u. Shapes of the two optimal controls $u_{1}$ and $u_{2}$ in Figure 8, do not change for both cases (a) " $k_{1}=0.9$, $k_{2}=0.9$ " and (b) " $k_{1}=0.69, k_{2}=0.69$ ", and that could recommend us to use a treatment against HDV infection for only 230 

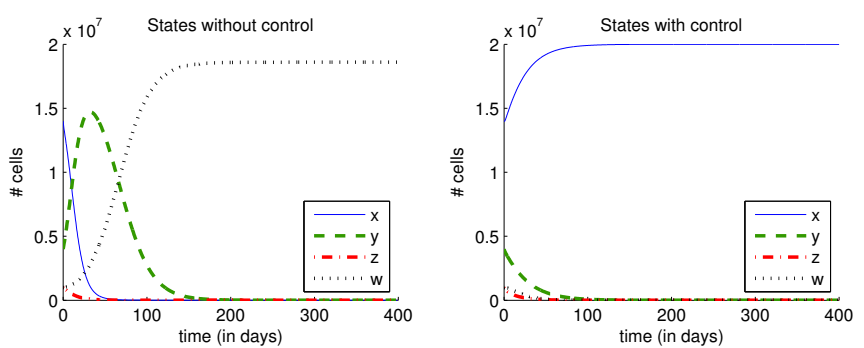

Fig. 9. Comparison between states $\mathrm{x}, \mathrm{y}, \mathrm{z}$ and $\mathrm{w}$ of system (12/15) before and after treatment with $R_{b}=3, R_{d}=0.6, k_{1}=0.9, k_{2}=0.9$
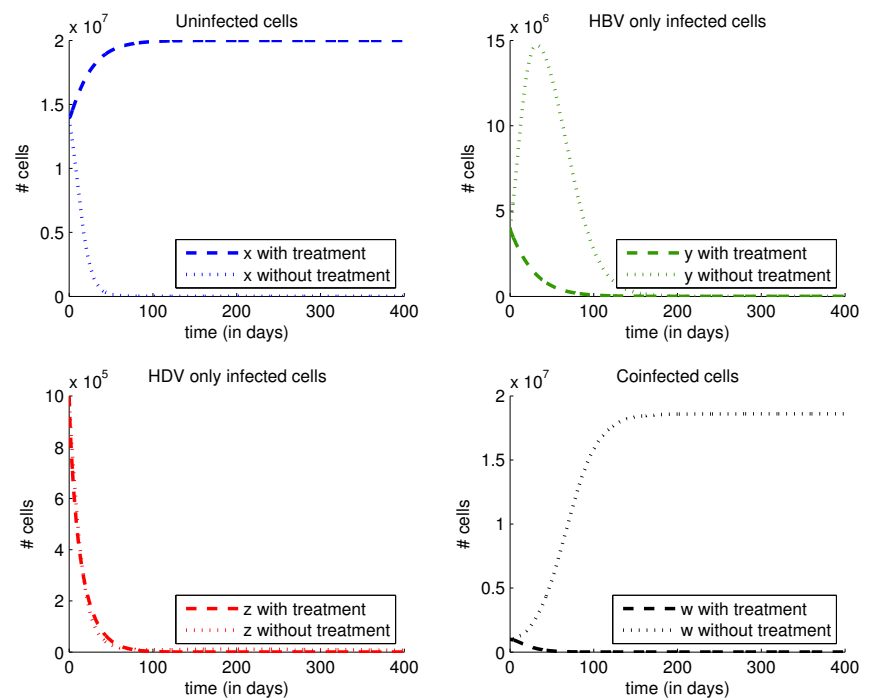

Fig. 10. Comparison between states $\mathrm{x}, \mathrm{y}, \mathrm{z}$ and $\mathrm{w}$ of system 12,15 before and after treatment with $R_{b}=3, R_{d}=0.6, k_{1}=0.9, k_{2}=0.9$
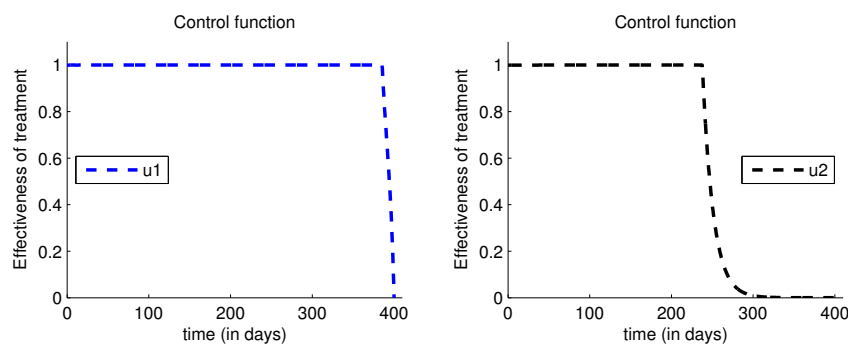

Fig. 11. Control functions with $\delta=0.051975, \sigma=0.2079 . k_{1}=0.9$, $k_{2}=0.9$.

days in the case of a small value of the reproduction number $R_{d}$, while following an antiviral therapy for HBV infection during 400 days because of the big value of the reproduction number $R_{b}$.

Third case : (Infected cell proliferation) $R_{b}=3, R_{d}=0.6$, $k_{1}=0.8, k_{2}=0.8$

For the numerical simulations associated to the second part of this article, and that concerns the model with infected cell proliferation, the values utilized for both $\mathrm{HBV}$ and HDV reproduction numbers were $R_{b}=3$ and $R_{d}=0.6$, where $R_{b}=\frac{\sigma}{a}$ and $R_{d}=\frac{\delta+c \sigma}{a}=\frac{\delta}{a}+c R_{b}$ [2]. In Figure 9, on left, and in Figure 10, (a)
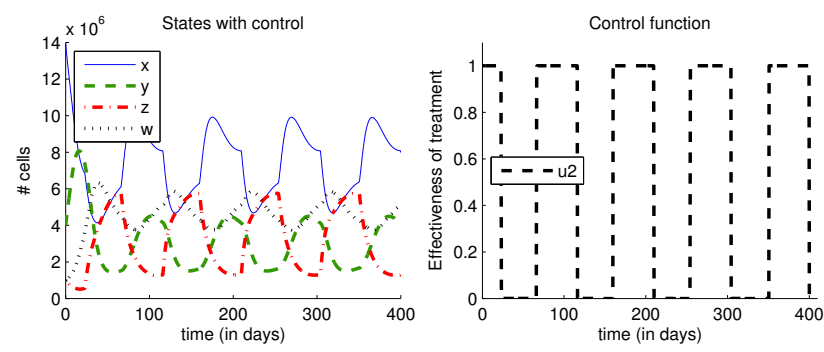

(b)
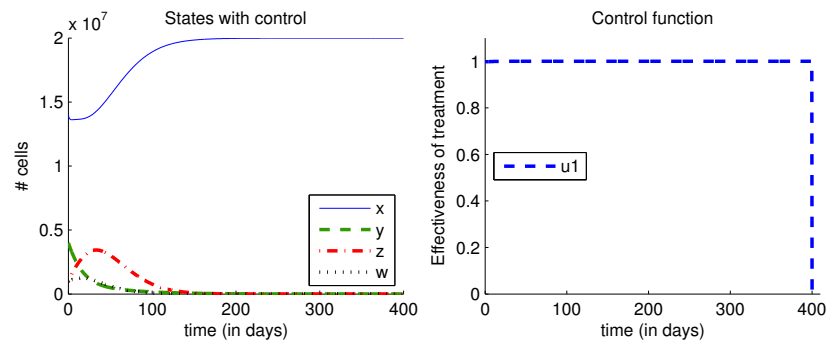

Fig. 12. States $\mathrm{x}, \mathrm{y}, \mathrm{z}$ and $\mathrm{w}$ of system 69 in the case of one control with $\delta=0.2772, \sigma=0.17325$. (a) $u_{1}=0, k_{2}=0.8$. (b) $u_{2}=0, k_{1}=0.8$.

(a)
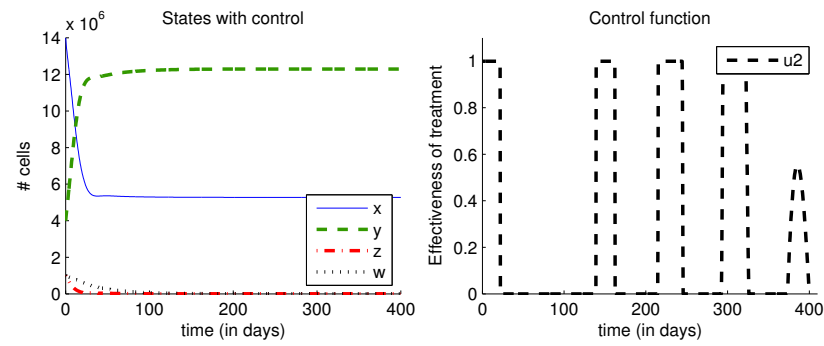

(b)
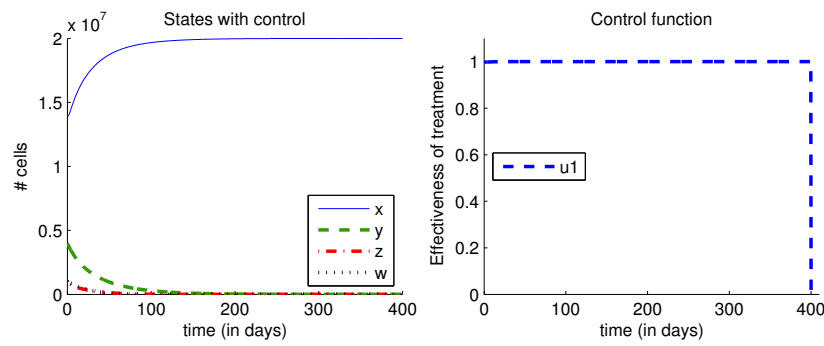

Fig. 13. States $\mathrm{x}, \mathrm{y}, \mathrm{z}$ and $\mathrm{w}$ of system 69 in the case of one control with $\delta=0.04158, \sigma=0.17325$. (a) $u_{1}=0, k_{2}=0.8$. (b) $u_{2}=0, k_{1}=0.8$.

dynamics between uninfected cells (x), HBV infected cells, HDV infected cells and coinfected cells in the absence of treatment, are observed, and Figures 11 and on right sides in Figures 12, 13 and 14 , it is observed simulations of the sought optimal controls represented by $u_{1}$ and $u_{2}$, and that represent the effectiveness of the treatments in the case of infected cell proliferation. $\mathrm{y}$ and $\mathrm{w}$ cells increase towards the values $1.5 \times 10^{6}$ c.f.u. and $1.9 \times 10^{6}$ c.f.u. respectively after 45 and 170 days of the infection period while $\mathrm{x}$ and w cells taper off after only 50 and 80 days. In Figure 9, on right, and in Figure 10, it is deduced that treatment has an effect in decreas- 

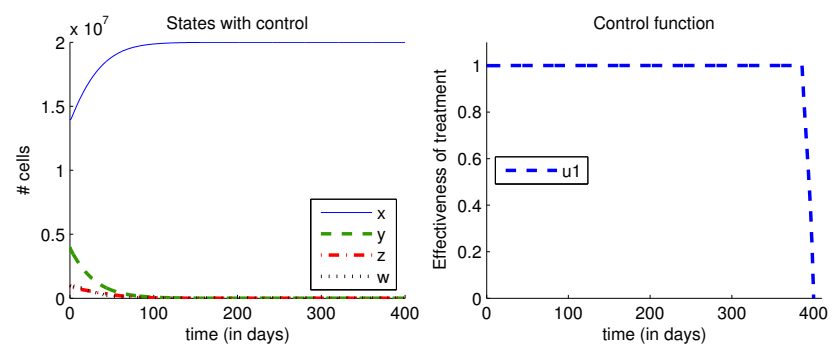

Fig. 14. States $\mathrm{x}, \mathrm{y}, \mathrm{z}$ and $\mathrm{w}$ of system 1215 in the case of one control with $\delta=0.51975, \sigma=0.2079 . u_{2}=0, k_{1}=0.9$.

ing the number of both HBV and HDV infected cells after only 100 days, and also coinfected cells after only 50 days. As regards to the optimal controls $u_{1}$ and $u_{2}$, they both conserve the value 1 until 390 and 260 days respectively, and that could be understood by recommending a reduction from the HBV-therapy period stopping it at the 390th day of treatment and from the HDV-therapy stopping it at the 260th day of treatment knowing the effectiveness of any treatment is obviously related to its dosage amount utilized, and then if a treatment is not effective in some periods of therapy, then there is no need to inject it anymore. There is no specific or effective treatment/vaccine against acute or chronic HDV infection, and many researchers reported immunotherapy using interferons could represent a possible approach to fight against hepatitis $\mathrm{D}$ and also for other hepatitis types [13, 21]. Numerical results of first cases (a) in Figures 12 and 13 for a model without infected cell proliferation, present good examples to verify mathematically that in case of the absence of the treatment against B viral infection " $u_{1}=0$ " and with $u_{2}$ representing effectiveness for HDV inhibition therapy for instance, could participate in a decrease of $\mathrm{z}$ and $\mathrm{w}$ cells associated to HDV and HBV-HDV coinfection respectively. In fact, in case (a) of Figure 12, it is deduced that when $u_{2}$ is zero for approximately one month, uninfected cells are decreasing, and infection related to $\mathrm{y}, \mathrm{z}$ and $\mathrm{w}$ cells, is important, but when $u_{2}=1$ for approximately one month, uninfected cells are maximized and infection begin to decrease. As regards the case (a) in the Figure 13 , because of a smaller value of $R_{d}$, a treatment against HDV and HBV-HDV coinfection, seems successful when $u_{2}=1$ in the first 20 days of the therapy, but because a rest period of about 110 days after, y cells gain a bigger cells and $\mathrm{x}$ cells taper to minimal value of about $5.5 \times 10^{6}$ c.f.u. However, Lyle et al. [1], Heidrich et al. [21] think immunization against HBV only, seems effective even in reducing the number of viruses Delta. In the cases (b) of Figures 12 and 13, and also in Figure 14 that is associated to the model with infected cell proliferation, it could be observed also that by an optimal control approach, affecting zero value to the second control $u_{2}$, was sufficient for the cure from the three types of infection when $u_{1}$ takes the value 1 .

\section{CONCLUSION}

Despite of the existence of treatments against HBV infection, there are still millions of chronically infected people worldwide, and treatments against HDV infection are not known yet. In this work, and based on Pontryagin's maximum principle, better treatment strategies were identified for HBV-HDV coinfection, when only one treatment is used against B viruses, and when only one treatment is used against Delta viruses, and when two treatments were used against both viruses B and Delta., in order to block new infection and prevent viral production by using a bitherapy with lesser side-effects. The numerical simulations obtained in this article, were very important in a way they were utilized to prove a coherence between the results of the optimal control approach, and the experimental results reported in studies of many doctors in the field, showing a possibility of the cure from HBV-HDV coinfection while following an optimal antiviral bitherapy.

\section{REFERENCES}

[1] Lyle, W., Hecht, A., Hepatitis (Deadly Diseases and Epidemics), Library Binding, September 2011. (2011)

[2] A.Packer, J.Forde, S.Hews, Y.Kuang, Mathematical models of the interrelated dynamics of hepatitis D and B, Mathematical Biosciences 247 (2014) 38-46.

[3] Hughes, S. A., Wedemeyer, H., \& Harrison, P. M. (2011). Hepatitis delta virus. The Lancet, 378(9785), 73-85.

[4] Wedemeyer, H., \& Manns, M. P. (2010). Epidemiology, pathogenesis and management of hepatitis D: update and challenges ahead. Nature Reviews Gastroenterology and Hepatology, 7(1), 31-40.

[5] James, H., Chow, Chow. C., Facts on File Library of Health and Living, The Encyclopedia of Hepatitis And Other Liver Diseases -Facts on File. (2006).

[6] Rizzetto, M., Canese, M. G., Arico, S., Crivelli, O., Trepo, C., Bonino, F., \& Verme, G. (1977). Immunofluorescence detection of new antigen-antibody system (delta/anti-delta) associated to hepatitis B virus in liver and in serum of HBsAg carriers. Gut, 18(12), 997-1003.

[7] Hiroshi, H., Yuki, Y., Medical Intelligence Unit, Hepatitis Delta Virus-Springer US. (2006).

[8] Purcell, R., The discovery of the hepatitis viruses. Gastroenterology 1993, 104:955-63. (1993)

[9] Rizzetto, M., Hoyer, B., Canese, M., et al. Delta antigen : the association of delta antigen with hepatitis B surface antigen and ribonucleic acid in serum of delta infected chimpanzees. Proc Natl Acad Sci USA 1980 , 77:6124-8. (1980).

[10] Rizzetto, M. (1983). The delta agent. Hepatology, 3(5), 729737.

[11] Chakrabarty, S. P., \& Joshi, H. R. (2009). Optimally controlled treatment strategy using interferon and ribavirin for hepatitis C. Journal of Biological Systems, 17(01), 97-110.

[12] Elmouki, I., \& Saadi, S. (2015). Quadratic and linear controls developing an optimal treatment for the use of BCG immunotherapy in superficial bladder cancer. Optimal Control Applications and Methods. doi:10.1002/oca.2161

[13] Iwamoto, M., Watashi, K., Tsukuda, S., Aly, H. H., Fukasawa, M., Fujimoto, A., ... \& Wakita, T. (2014). Evaluation and identification of hepatitis B virus entry inhibitors using HepG2 cells overexpressing a membrane transporter NTCP. Biochemical and biophysical research communications, 443(3), 808-813.

[14] Lempp, F. A., \& Urban, S. (2014). Inhibitors of hepatitis B virus attachment and entry. Intervirology, 57(3-4), 151-157.

[15] Heidrich, B., Manns, M. P., \& Wedemeyer, H. (2013). Treatment options for hepatitis delta virus infection. Current infectious disease reports, 15(1), 31-38.

[16] Rizzetto, Mario, and Alessia Ciancio. The prenylation inhibitor, lonafarnib: a new therapeutic strategy against hepatitis delta. The Lancet Infectious Diseases (2015). 
[17] Koh, Christopher, et al. Prenylation inhibition with lonafarnib decreases hepatitis D levels in humans. The Liver Meeting. 2014.

[18] Koh, Christopher, et al. Oral prenylation inhibition with lonafarnib in chronic hepatitis D infection: a proof-of-concept randomised, double-blind, placebo-controlled phase $2 \mathrm{~A}$ trial. The Lancet Infectious Diseases (2015).

[19] Fister KR, Panetta JC (2003) Optimal control applied to competing chemotherapeutic cell-kill strategies. SIAM J Appl Math 63:1954

[20] Almeida, JuneD, D. Rubenstein, and E. J. Stott. New antigenantibody system in Australia-antigen-positive hepatitis. The Lancet 298.7736 (1971): 1225-1227.

[21] Farci, P., Mandas, A., Coiana, A., Lai, M. E., Desmet, V., Van Eyken, P., ... \& Balestrieri, A. (1994). Treatment of chronic hepatitis D with interferon alfa-2a. New England Journal of Medicine, 330(2), 88-94.

[22] Weltman, M. D., Brotodihardjo, A., Crewe, E. B., Farrell, G. C., Bililus, M., Grierson, J. M., \& Liddle, C. (1995). Coinfection with hepatitis $\mathrm{B}$ and $\mathrm{C}$ or $\mathrm{B}, \mathrm{C}$ and $\mathrm{d}$ viruses results in severe chronic liver disease and responds poorly to terferon-a treatment. Journal of viral hepatitis, 2(1), 39-45.

[23] Joshi, H. R. (2002). Optimal control of an HIV immunology model. Optimal control applications and methods, 23(4), 199213.

[24] L. S. Pontryagin, V. G. Boltyanskii, R. V. Gamkrelidze, E. F. Mishchenko, The Mathematical Theory of Optimal Processes, Wiley, New York, 1962.

[25] Lenhart, S., \& Workman, J. T. (2007). Optimal control applied to biological models. CRC Press.

[26] Jung, E., Lenhart, S., \& Feng, Z. (2002). Optimal control of treatments in a two-strain tuberculosis model. Discrete and Continuous Dynamical Systems Series B, 2(4), 473-482. 